\title{
Search for Two-Scale Localization in Disordered Wires in a Magnetic Field
}

\author{
$\mathrm{H}$ Schomerus and C W J Beenakke1 \\ Instituut Lorentz, Untversitelt Leiden PO Box 95062300 RA Letden The Netherlands
}

(Recerved 1 Novembel 1999)

\begin{abstract}
A recent paper [A V Kolesnikov and K B Efetov, Phys Rev Lett 83, 3689 (1999)] predicts a two-scale behavior of wave function decay in disordered wires in the crossover regime from preserved to broken time reversal symmetry We have tested this piediction by a transmission approach, relying on the Borland conjecture that relates the decay length of the tiansmittance to the decay length of the wave functions Our numerical simulations show no indication of two scale behavior
\end{abstract}

PACS numbe1s $7215 \mathrm{Rn} 0560 \mathrm{Gg}, 7320 \mathrm{Fz}$

In a 1emaikable pape1 [1], Kolesnikov and Efetov have piedicted that the decay of wave functions in disordered wites is chaiacterized by two localization lengths, if timereversal symmetry is pattially bioken by a weak magnetic field Using the supersymmetry technique [2], it was demonstrated that the far tarl of the wave functions decays with the length $\xi_{2}$ characteristic for completely bioken time-1 eversal symmetiy - even if the flux through a localized area is much smaller than a flux quantum At shoiter distances the decay length is $\xi_{1}=\frac{1}{2} \xi_{2}$ It was suspected that previous studies by Pichard et al [3] found single-scale decay because of the misguiding theoretical expectation of such behavior This expectation was also the basis for the interpretation of the experiments by Khavin, Gershenson, and Bogdanov [4] on submicron-wide wires

The prediction of Kolesnikov and Efetov calls for a test by means of a dedicated experiment or computer simulation It is the purpose of this work to provide the latter We target the key feature of the two-scale localization phenomenon, which is the doubling of the asymptotic decay length at infinitesımally weak magnetıc fields

Our numerical simulations are based on a transmission appioach We rely on the Borland conjecture [5] (believed to be true geneially [6]) that relates the asymptotic decay of the transmittance $T$ with increasing wire length $L$ to the asymptotic decay of the wave function $\psi(L)$ According to the Borland conjecture, the Lyapunov exponent $\alpha=-\lim _{L \rightarrow \infty} \frac{1}{2} L^{-1} \ln T$ is identical to the inverse localization length $\xi^{-1}=-\lim _{L \rightarrow \infty} L^{-1} \ln |\psi(L)|$ Moreover, $\xi$ and $\alpha$ are self-averaging, meaning that the statistical fluctuations become smaller and smaller as $L \rightarrow \infty$ Our numerical simulations show that the ciossover from $\xi=\xi_{1}$ to $\xi=\xi_{2}$ does not occui unt1l the flux $\Phi_{\xi}$ through a wile segment of length $\xi_{1}$ is of the order of a flux quantum $\Phi_{0}=h / e$ For our longest wires $\left(L \geqslant 150 \xi_{1}\right)$, the crossover according to Ref [1] should have occurred at $\Phi_{\xi} / \Phi_{0} \simeq \exp \left(-L / 8 \xi_{1}\right) \simeq 10^{-8}$ We consider various possible reasons for the disagreement, and suggest that the quantity considered in Ref [1] is dominated by anomalously localızed states

Our first set of 1esults is obtained from the numerical calculation (by the technique of recursive Gieen functions
[7]) of the tiansmission matrix $t$ for a two-dimensional Anderson Hamiltonian with on-site disor der In units of the lattice constant $a \equiv 1$, the width of the wire is $W=13$ and the wavelength of the electrons is $\lambda=51$, 1esulting in $N=5$ propagating modes thiough the wire The localization lengths $\xi_{1}=(N+1) l$ and $\xi_{2}=2 N l$ ate determined by the scaling paiameter $l$ of quasi-one-dimensional localization theory, which differs from the transport mean-fiee path by a coefficient of or der unity [8] The average of the transmittance $T=\mathrm{t} t t^{\dagger}$ in the metallic iegime, fitted to $\langle T\rangle=N(1+L / l)^{-1}$, yields $l=65$ This gives a local1zation length $\xi_{1}=390$ for preserved time-1eveisal symmetiy (symmetry index $\beta=1$ ) and a localization length $\xi_{2}=650$ for broken time-reversal symmetiy $(\beta=2)$

Figure 1 shows the ensemble-averaged logarithm of the transmittance $\langle\ln T\rangle$ as a function of wire length $L$ for var1ous values of the magnetic field $B$ (or flux $\Phi_{\xi}=W \xi_{1} B$ ) We find a smooth tiansition between the theoretical expectations for pieserved and broken time-reversal symmetiy Most impoitantly, we find an asymptotic slope $s(B)=\lim _{L \rightarrow \infty} L^{-1}\langle\ln T\rangle$ that interpolates smoothly between the values $s=-2 / \xi_{1}$ for $B=0$ and $s=-2 / \xi_{2}$ for large $B$ There is no indication of a crossover to the slope $s=-2 / \xi_{2}$ for smaller values of $B$, even for very long wires $\left(L \geq 150 \xi_{1}\right)$ According to the theory of Ref [1], the crossover should occur at a length $L_{\text {cross }}$ given by

$$
L_{\text {cross }} / \xi_{1}=8 \ln \left(\sqrt{12} \Phi_{0} / 4 \pi \Phi_{\xi}\right)+\mathcal{O}(1),
$$

which is well within the range of our simulations $\left(L_{\text {cross }} \simeq\right.$ $14 \xi_{1}$ for $\Phi_{\xi} \simeq 005 \Phi_{0}$ ) The absence of two-scale behav1or in the transmittance of an individual, arbitrarily chosen realization is demonstrated in the inset of Fig 1 , for $\Phi_{\xi}=\frac{1}{2} \Phi_{0}$ The self-aver aging property of the Lyapunov exponent is evident

The asymptotic decay length $\xi(B)=-2 / s(B)$ is plotted veisus magnetic field in Fig 2 , together with the weaklocalization conection $\delta T=T(B=\infty)-T(B)$ at $L=$ $\xi_{1}$ For both quantities, breaking of time-reversal symmet1y sets in when $\Phi_{\xi}$ is comparable to $\Phi_{0}$ The transition from $\beta=1$ to $\beta=2$ is completed for $\Phi_{\xi} \approx 100 \Phi_{0}$ 


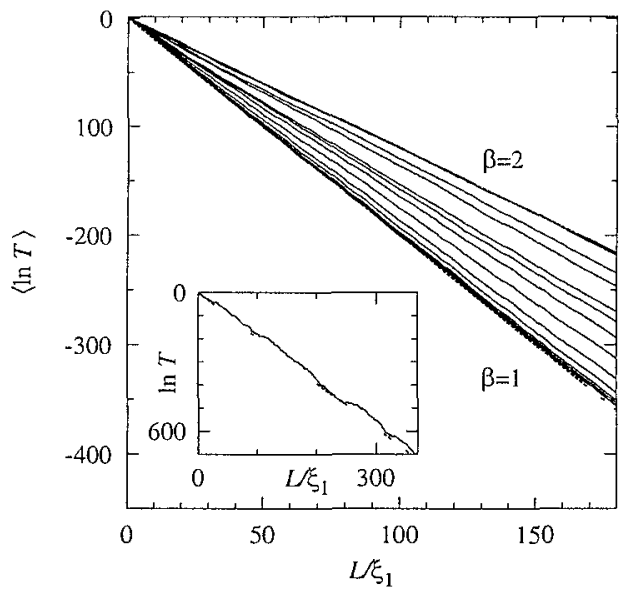

FIG 1 Average loganthmic transmittance $\langle\ln T\rangle$ as a function of wire length $L$ for the Anderson model with $N=5$ propagating modes The two dashed lines have the slopes piedicted for pieserved $(\beta=1)$ and broken $(\beta=2)$ time-i eversal symmetry From bottom to top the data correspond to fluxes $\Phi_{\xi} / \Phi_{0}=0$, 0 0005, 0 005, 005 (four indistınguishable solid curves), 05,1 , $25,5,10,15,20,25,40,50,75,125$ (two indistinguishable solid curves) The inset shows $\ln T$ for an individual realization with $\Phi_{\xi}=\frac{1}{2} \Phi_{0}$ (solid curve) and the slope of the ensembleaveraged result (dashed line) The statistical error is of the order of the wiggles of the curves

Our second set of results is obtained from a computationally more efficient model of a disordered wire, consisting of a chain of chaotic cavities (or quantum dots) with two leads attached on each side This so-called "domino" model [9] is similar to Efetov's model of a granulated metal [2] and to the Irda-Weidenmuller-Zuk model of connected slices [10] The length $L$ is now measured in units of cavities, and the mean-free path $l=1$ The scattering matrices of each cavity are randomly drawn from an ensemble (proposed by Zyczkowskı and Kuś [11]) that interpolates (by means of a parameter $\delta$ ) between the circular orthogonal $(\beta=1, \delta=0)$ and unitary $(\beta=2, \delta=$

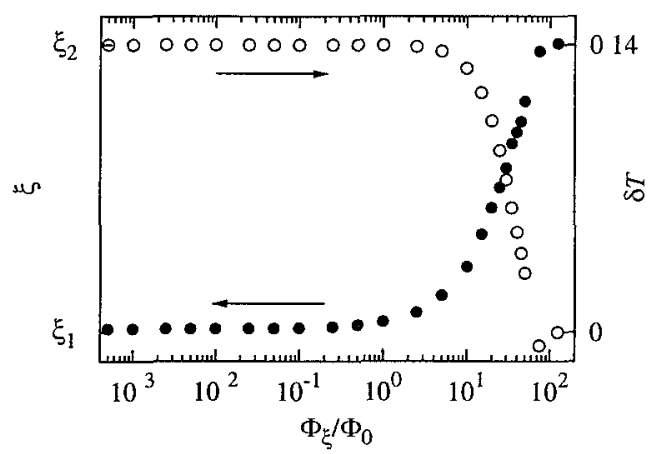

FIG 2 Asymptotic decay length (solnd circles) and weaklocalization correction $\delta T$ (open circles) as a function of flux for the $N=5$ Anderson model The statistical error is of the order of the size of the circles
1) ensembles of random-matix theory The relationship between $\delta$ and $\Phi_{\xi} / \Phi_{0}$ is linear for $\delta \ll 1$

We increased the number of propagating modes to $N=$ 50 , because it is concervable that the two-scale localization becomes manifest only in the large $N$ limit, or that only in this limit the critical flux $\Phi_{\xi}$ for the tiansition from $\xi_{1}$ to $\xi_{2}$ becomes $\ll \Phi_{0}$ (In the experıments of Ref [4] $N \approx$ 10 , so our simulations are in the experimentally relevant range of $N$ ) Because of the much larger value of $N$, we restricted ourselves for larger values of the magnetic flux to $L \simeq 25 \xi_{1}$, which should be sufficient to observe the local1zation length $\xi_{2}$ for $\Phi_{\xi} / \Phi_{0} \geqslant 10^{-2}$ For smallev values of the flux, we increased the wire length to $L \approx 100 \xi_{1}$ The data are presented in Fig 3 It is qualitatively similar to the results for the $N=5$ Anderson model Instead of two-scale behavior, we see only a single decay length which crosses over smoothly fiom $\xi_{1}$ to $\xi_{2}$ with incieasing $\delta$ Again, the crossover of $\xi$ coincides with the crossover of the weak-localization correction, so there is no anomalously small c1ossover flux for the localization length

The logarithmic average $\langle\ln T\rangle$ is the experimentally relevant quantity since it is representative for a single realization (see Fig 1, inset) The average transmittance
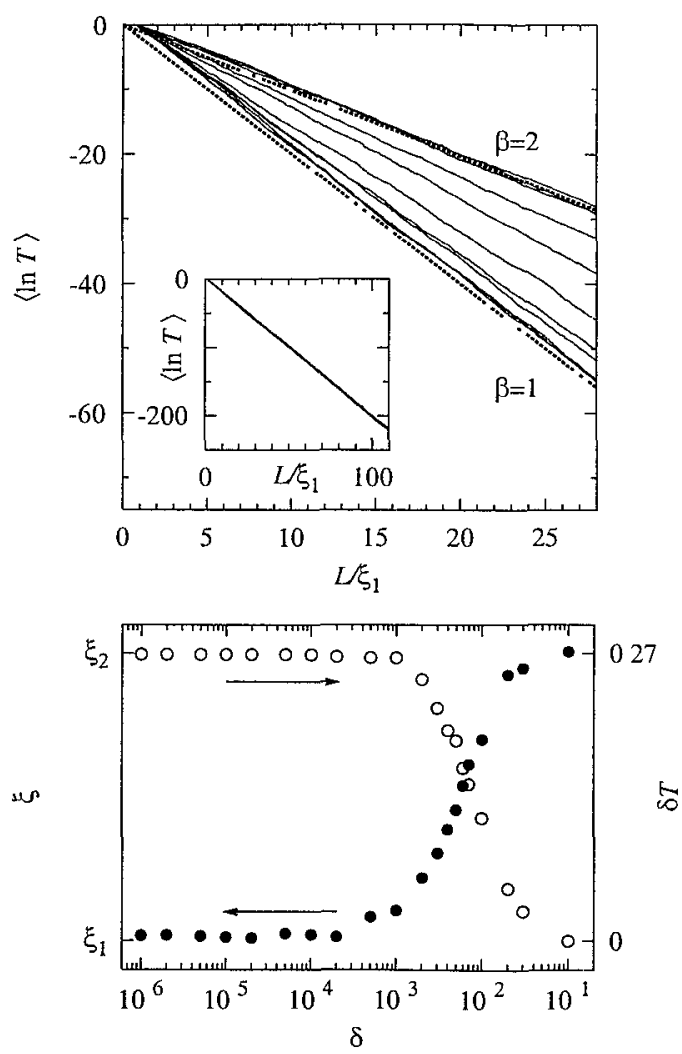

FIG 3 Same quantities as in Figs 1 and 2, but now for the $N=50$ domino model In the upper panel, the magnetic flux parameter $\delta=0,00001,00002,00005,0001,0002,0005$, $001,002,005$, and 01 In the inset, $\delta=0,000001$, and 00001 (indistınguishable curves) 


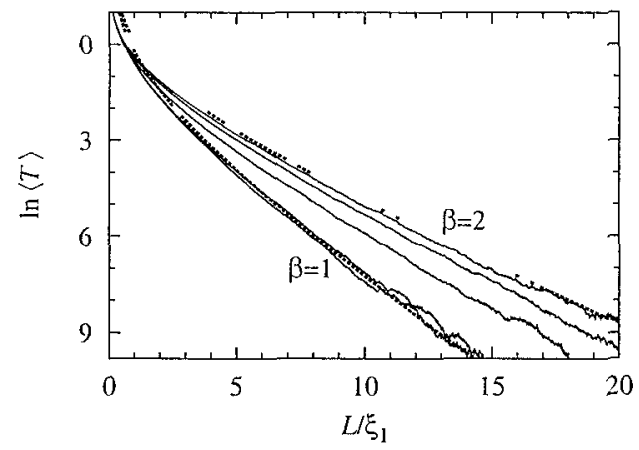

FIG 4 Logarithm of the average transmittance $\ln \langle T\rangle$ as a function of wire length $L$ for the $N=5$ Anderson model at various values of the magnetic field (solid curves, from bottom to top, $\Phi_{\xi} / \Phi_{0}=0,5,25,50,125$ ) The dashed curves ale the theoretical prediction of Refs $[13,14]$ for zero and la1ge magnetic field

$\langle T\rangle$ itself is not representative, because it is dominated by rare occurrences of anomalously localized states [12] Since Kolesnikov and Efetov [1] studied the average of wave functions themselves, 1ather than the average of logarithms of wave functions, it is conceivable that their findings are the iesult of such rare occurrences For completely broken or fully preserved time-reveisal symmetiy the average transmittance is given by [13]

$$
\ln \langle T\rangle=-L / 2 \xi_{\beta}-\frac{3}{2} \ln L / \xi_{\beta}+\mathcal{O}(1)
$$

The order 1 terms are also known $[13,14]$ and contilbute significantly for $L \leqq 30 \xi_{1}$ (This is the numerically accessible range, because anomalously localized states become exponentially rare with increasing wire length) We have plotted the full expressions in $\mathrm{F}_{1} g$ (dashed curves), together with the numerical data for the $N=5$ Anderson model Again we find a smooth crossover between preseived and broken time-reversal symmetry There is no transition with increasing wire length to a behavior indicative of completely broken time-reversal symmetry, even though the flux $\Phi_{\xi}$ is much larger than required [according to Eq (1)] to observe this crossover for the wave functions

In conclusion, we have piesented a numerical search for the two-scale localization phenomenon predicted by Kolesnikov and Efetov [1], with a negative result The asymptotic decay length of the transmittance is found to be given by $\xi_{1}$ and not by $\xi_{2}$, as long as the flux through a localization atea is small compared to the flux quantum How can one reconcile this numerical finding with the result of the supersymmetry theory? We give three possibilities (1) One might abandon the Borland conjecture and peimit the asymptotic decay length of the transmittance (Lyapunov exponent) to differ fiom the asymptotic decay length of the wave function (Iocalization length) Since the Borland conjecture has been the colneistone of localization theory for more than thi ee decades, this seems a too drastic solution (11) One could argue that the wires in the simulation are too nailow or too short - although they are in the experimentally relevant range of $N$ and $L$, as well as in the range of applicability of the theory of Ref [1] (111) One could attiıbute the two-scale localization phenomenon to anomalously localized states that are almost fully transmitted but become exponentrally iare with increasing length and are irrelevant for a typical wire This seems to be the most likely solution The decay due to anomalously localized states is solely due to their exponentrally decreasing fiaction among all states, and is not directly related to the localization length For the limiting cases of fully pieseived or totally bioken time-reversal symmetry, the decay is by a factor of 4 slower than the localızation length, but a two-scale behavior for partially bioken time-reversal symmetry is concervable

A discussion with P G Silvestrov motivated us to look into this pioblem We acknowledge helpful correspondence with A V Kolesnikov and support by the Dutch Science Foundation NWO/FOM

[1] A V Kolesnikov and K B Efetov, Phys Rev Lett 83, 3689 (1999)

[2] K B Efetov, Supersymmetry in Disoider and Chaos (Cambildge University, Cambridge, England, 1997)

[3] J -L P1chard, M Sanquer, K Slevin, and P Debray, Phys Rev Lett 65, 1812 (1990)

[4] Yu B Khavin, M E Gershenson, and A L Bogdanov, Phys Rev Lett 81, 1066 (1998), Phys Rev B 58, 8009 (1998)

[5] R E Borland, Proc R Soc London A 274, 529 (1963)

[6] A Ci1sant1, G Paladin, and A Vulpran1, Products of Random Matrices (Springer-Verlag, Betlın, 1993)

[7] H U Baranger, D P DiVincenzo, R A Jalabert, and A D Stone, Phys Rev B 44, 10637 (1991)

[8] C W J Beenakker, Rev Mod Phys 69, 731 (1997)

[9] T Dittrich, E Doron, and U Smulansky, J Phys A 27, 79 (1994)

[10] S Iida, H A Weidenmuller, and J A Zuk, Ann Phys (N Y) 200, 219 (1990)

[11] K Zyczkowsk1 and M Kuś, Phys Rev E 53, 319 (1996)

[12] A D Mrrlin, Phys Rep 326, 259 (2000)

[13] M R Zirnbauer, Phys Rev Lett 69, 1584 (1992)

[14] A D Mrrin, A Muller-Groelıng, and M R Zirnbauer, Ann Phys (N Y) 236, 325 (1994) 